\title{
Nanodiamond Theranostic for Light-Controlled Intracellular Heating and Nanoscale Temperature Sensing
}

Yingke Wu, $\uparrow$ Md Noor A Alam, $\uparrow$ Priyadharshini Balasubramanian, Anna Ermakova, Stephan Fischer, Holger Barth, Manfred Wagner, Marco Raabe, ${ }^{*}$ Fedor Jelezko, * and Tanja Weil*

Y. Wu, M. N. A. Alam, Dr. A. Ermakova, Dr. M. Wagner, M. Raabe, Prof. Dr. T. Weil

Max Planck Institute for Polymer Research, Ackermannweg 10, 55128 Mainz, Germany

*E-mail: weil@mpip-mainz.mpg.de, raabe@mpip-mainz.mpg.de

Y. Wu, M. N. A. Alam, M. Raabe, Prof. Dr. T. Weil

Institute of Inorganic Chemistry I, Ulm University, Albert-Einstein-Allee 11, $89081 \mathrm{Ulm}$, Germany

P. Balasubramanian, Prof. Dr. F. Jelezko

Institute for Quantum Optics, Ulm University, Albert-Einstein-Allee 11, 89081 Ulm, Germany

*E-mail: fedor.jelezko@uni-ulm.de

Dr. S. Fischer, Prof. Dr. H. Barth

Institute of Pharmacology and Toxicology, University of Ulm Medical Center, $89081 \mathrm{Ulm}$, Germany

Keywords: Nanodiamond, nanogel, intracellular temperature manipulation and sensing, photothermal application.

\begin{abstract}
Temperature is an essential parameter in all biological systems but information about the actual temperature in living cells is limited. Especially, in photothermal therapy, local intracellular temperature changes induce cell death but the local temperature gradients are not known. Highly sensitive nanothermometers would be required to measure and report local temperature changes independent of the intracellular environment including $\mathrm{pH}$ or ions. Fluorescent
\end{abstract}


nanodiamonds (ND) enable temperature sensing at the nanoscale independent of external conditions. Herein, we prepare ND nanothermometers coated with a nanogel shell and the photothermal agent indocyanine green that serves as a heat generator and sensor. Upon irradiation, programmed cell death was induced in cancer cells with high spatial control. In parallel, the increase in local temperature was recorded by the NDs nanothermometers. This approach represents a great step forward to record local temperature changes in different cellular environments inside cells and correlate these with thermal biology.

\section{Main}

Temperature plays a fundamental role in biological processes of living organisms and is involved in cell differentiation, proliferation, and death ${ }^{1}$, protein functions ${ }^{2,3}$ as well as gene expression. ${ }^{4}$ Hence, probing or even manipulating the local intracellular temperature with high spatial control is crucial to understand the fundamental relationship between biological activities and temperature. Cells are very sensitive to temperature changes, which is exploited in photothermal therapy (PTT), where tumor cells $\mathrm{s}^{5,6}$ or even the entire tumors ${ }^{7-9}$ are eliminated or shrink if the intracellular temperature exceeded $42{ }^{\circ} \mathrm{C}$ or even $50{ }^{\circ} \mathrm{C}$, respectively. Counterintuitively, cell organelles such as mitochondria maintain a physiological temperature of close to $50{ }^{\circ} \mathrm{C}$ without harming the cell. ${ }^{10}$ Therefore, there is a fundamental interest to obtain a deeper understanding of the local intracellular temperature as well as temperature changes during therapy and the impact on biological systems.

PTT is routinely used in patients to increase the local temperature in diseased cells or tissues and it is effective in the treatment of certain cancers. ${ }^{5,11}$ During PTT, a photothermal agent (PA) is delivered into tumor tissue and upon illumination, the PA converts absorbed light energy 
into heat. Over time, this process leads to either partial or complete ablation of the tumor tissue. ${ }^{12}$ Various PAs have been designed, mainly in the form of nanomaterials, which benefit from the enhanced permeability and retention (EPR) effect of tumor tissue after intravenous injection $^{13,14}$. However, these PAs lack the ability to sense and report local temperature changes and it is not possible to correlate the induced local temperature and the impact on apoptosis. To improve the efficacy of PTT based on rational information, the relationship between local temperature changes and induced controlled cell death via apoptosis would be crucial.

Different kinds of fluorescence-based thermometer have been explored to directly measure the intracellular temperature at the nanoscale. ${ }^{15-17}$ However, environmental parameters like $\mathrm{pH}$, ion concentrations or intracellular viscosity could affect their sensitivity resulting in inaccurate temperature readout. ${ }^{18}$ Nanodiamonds (NDs) containing negatively charged nitrogen-vacancy $\left(\mathrm{NV}^{-}\right)$centers show neither photoblinking nor photobleaching ${ }^{19}$ and their fluorescence is hardly influenced by $\mathrm{pH}$, ion concentration, viscosity, molecular interactions or organic solvents. ${ }^{20}$ The temperature response of NDs differs strongly from most fluorescence probes as the $\mathrm{NV}^{-}$ center reveals a thermal shift of the zero field splitting $\left(D_{0}\right)$ at $2.87 \mathrm{GHz}\left(\mathrm{m}_{\mathrm{s}}=0 \text { to } \mathrm{m}_{\mathrm{s}}= \pm 1\right)^{21-}$ ${ }^{23}$ or the zero-phonon line (ZPL) at $637 \mathrm{~nm} \cdot{ }^{24,25}$ Thus, NDs could serve as highly sensitive nanoscale thermometers in biological systems.

Herein, we report the preparation and application of an intracellular local heat generator and nanothermometer, termed nanodiamond-nanogel-indocyanine green (ND-NG-ICG), serving as an intracellular self-reporting photothermal system to probe the local temperature changes during PTT. Upon irradiation, programmed cell death (apoptosis) was induced in cancer cells with high spatial control. In parallel, local temperature increase was recorded inside endosomal vesicles of cancer cells. Our approach paves the way to ultimately optimize PTT based on quantitative information. Moreover, we see great prospects to explore the impact of intracellular temperature changes on cellular processes in thermal biology. 


\section{Preparation and characterization of ND-NG-ICG}

NDs need to be stabilized by a biocompatible surface coating to prevent aggregation in biological media and impart bioactive surface groups. We have recently reported the stabilization of NDs in a crosslinked nanogel ND-NG that were non-toxic in cell experiments. ${ }^{26}$ Hyperbranched polyethyleneimine (PEI) was used to precoated NDs in the presence of polyvinylpyrrolidone (PVP) as stabilizer, and subsequently, a 4-arm polyethyleneglycol $\mathrm{N}$ hydroxysuccinimid (NHS) ester was added to crosslink the precoated PEI on the surface of NDs in phosphate-buffered saline (PBS) buffer to form a stable, positively charged nanogel shell. Herein, we have adsorbed the anionic indocyanine green (ICG) by electrostatic interactions to yield ND-NG-ICG. Free ICG was removed from ND-NG-ICG by centrifugation $(12,000 \mathrm{rpm}$, Figure 1A). The amount of ICG, which was loaded onto the ND-NG has a great impact on the efficiency of PTT. Therefore, the concentration of free ICG in the supernatant after centrifugation was quantified by measuring its characteristic absorbance at $789 \mathrm{~nm}$. In this way, we avoid any interference of this method by the emission of the ND-NGs. $64.5 \mu \mathrm{g}$ ICG was loaded onto $200 \mu \mathrm{g}$ ND-NG, which corresponds to a loading efficiency of $24.4 \mathrm{wt} \%$ (Figure S1). In order to assess potential ICG leakage, the release of ICG was detected over time. Obviously, ND-NG-ICG remained stable and only about $10 \%$ ICG was released over 20 days (Figure S2). Since the hydrodynamic radius of nanoparticles greatly affect their cellular uptake, dynamic light scattering (DLS; Figure 1B) and transmission electron microscopy (TEM; Figure 1C and 1D) were accomplished to investigate the size distribution, shape and, morphology of ND-NG-ICG. In Milli-Q water, the hydrodynamic diameter of ND, ND-NG, and ND-NG-ICG increased from $37.7 \pm 0.23 \mathrm{~nm}, 56.9 \pm 0.27 \mathrm{~nm}$ to $99.6 \pm 0.53 \mathrm{~nm}$, respectively (Figure $\mathrm{S} 3$ ). ND-NG-ICG revealed a monomodal size distribution (Figure 1B) with a polydispersity index (PDI) of 0.243 and no aggregate formation was observed in solution. Also TEM image of ND- 
NG-ICG analysis showed individual nanoparticles without any obvious aggregation (Figure $1 \mathrm{C})$.

The photophysical properties of NDs containing $\mathrm{NV}^{-}$centers are essential for the application of ND-NG-ICG in bioimaging and intracellular temperature sensing. The $\mathrm{NV}^{-}$centers in NDs are very sensitive to surface charge and they can switch to the dark state (positively charged $\mathrm{NV}$ center; $\mathrm{NV}^{+}$) under certain conditions und in this case, they could not be used for sensing anymore. To further study the influence of the polymer coating on the $\mathrm{NV}^{-}$centers in NDs, ND-NG-ICG was dropped on a glass coverslip and spectra were recorded on a custom-built confocal microscope using a $532 \mathrm{~nm}$ excitation with a power of $110 \mu \mathrm{W}$ in front of the objective (oil, NA = 1.35). Upon irradiation of ND-NG-ICG at $532 \mathrm{~nm}$, the absorption spectrum of NDNG-ICG revealed slight decrease of the intensity of the peak at $680 \mathrm{~nm}$ due to a minor energy transfer component from ND to ICG because of partial overlap of the emission spectra of ND and the absorption spectra of ICG. The zero-phonon line of $\mathrm{NV}^{-}$at $637 \mathrm{~nm}$ was clearly visible without any background noise (Figure 1E) indicating that the $\mathrm{NV}^{-}$centers in ND-NG-ICG remained in the optically active state, which is necessary for intracellular temperature sensing. 

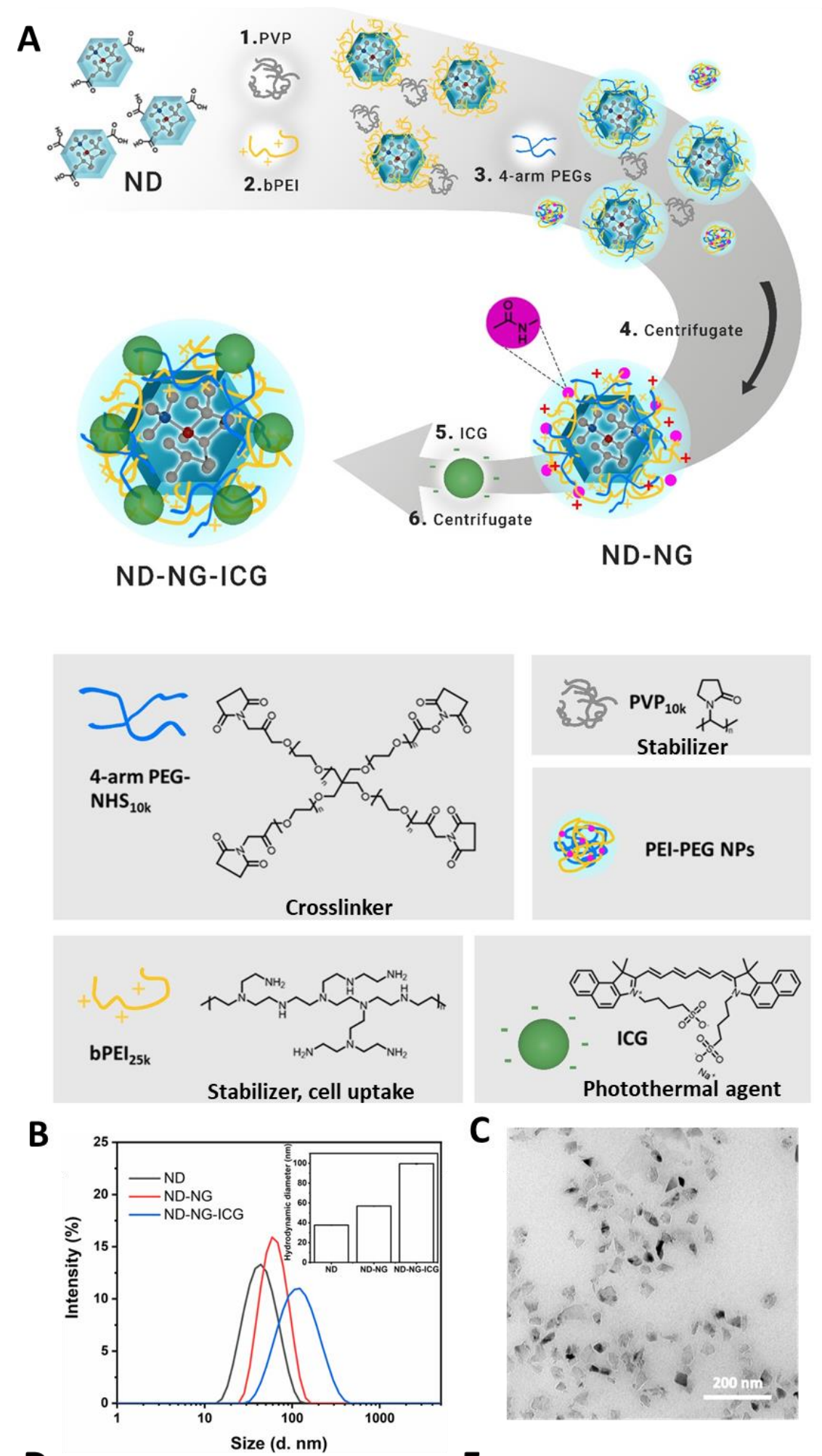

D
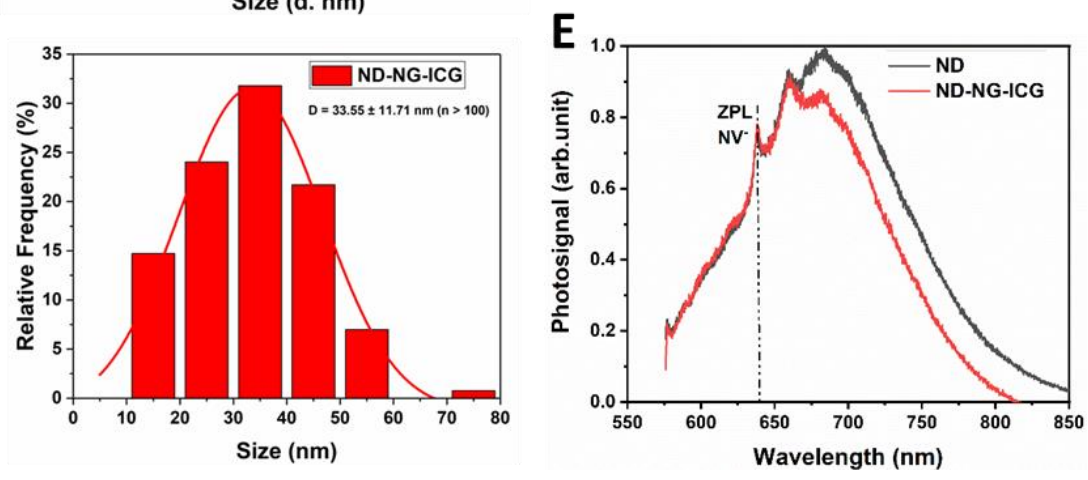
Figure 1. (A) Schematic illustration of the synthesis route of ND-NG-ICG. (B) The hydrodynamic diameter of ND, ND-NG, and ND-NG-ICG measured by DLS. (C) TEM images of ND-NG-ICG (scale bar = $200 \mathrm{~nm})$. (D) Histogram analysis of ND-NG-ICG $(\mathrm{n}>100)$. (E) Normalized emission spectra (ex. $532 \mathrm{~nm}$ ) of ND and ND-NG-ICG. The zero-phonon line (ZPL) of $\mathrm{NV}^{-}$is visible in both spectra.

\section{ND-NG-ICG reveal cell compatibility and intracellular localization inside endosomes}

The cell compatibility of ND-NG-ICG was investigated in a human cervical carcinoma cell line (HeLa). As displayed in Figure 2A, ND-NG-ICG showed high cell viability even after cell treatment using very high concentrations of up to $400 \mu \mathrm{g} / \mathrm{mL}$. In addition, as depicted in Figure 2B, ND-NG-ICG was efficiently taken up into HeLa cells and cell growth as well as cell morphology remained unaltered. The intracellular localization of ND-NG-ICG was imaged after incubating HeLa cell for 4 hours with two different nanoparticle concentrations, $10 \mu \mathrm{g} / \mathrm{mL}$ and $100 \mu \mathrm{g} / \mathrm{mL}$. Afterwards, cells were processed for TEM measurement (see details in method part, Figure 2C and 2D). We found internalized ND-NG-ICGs that were encapsulated in endosomal vesicles independent of the applied nanoparticle concentration indicating no significant difference in the cell uptake mechanism between $10 \mu \mathrm{g} / \mathrm{mL}$ and $100 \mu \mathrm{g} / \mathrm{mL}$ of NDNG-ICG. At lower concentrations (10 $\mu \mathrm{g} / \mathrm{mL}$ ND-NG-ICG), less vesicles containing ND-NGICG were found in cells. 
A

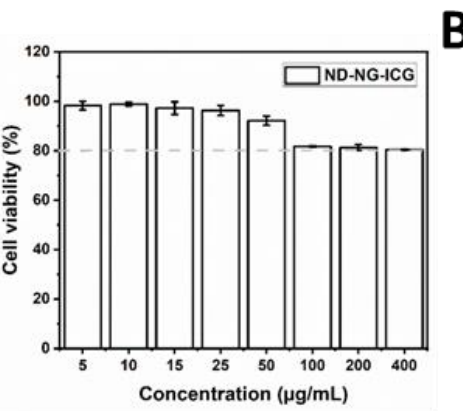

C

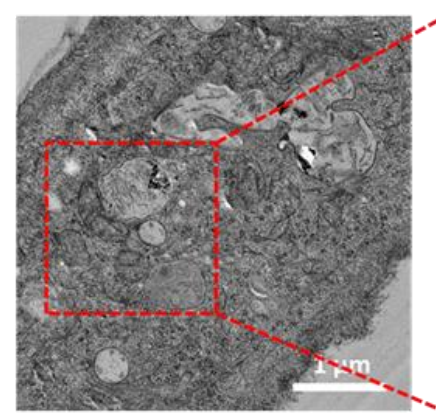

D

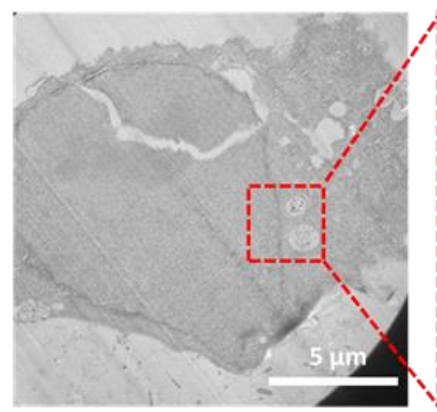

B
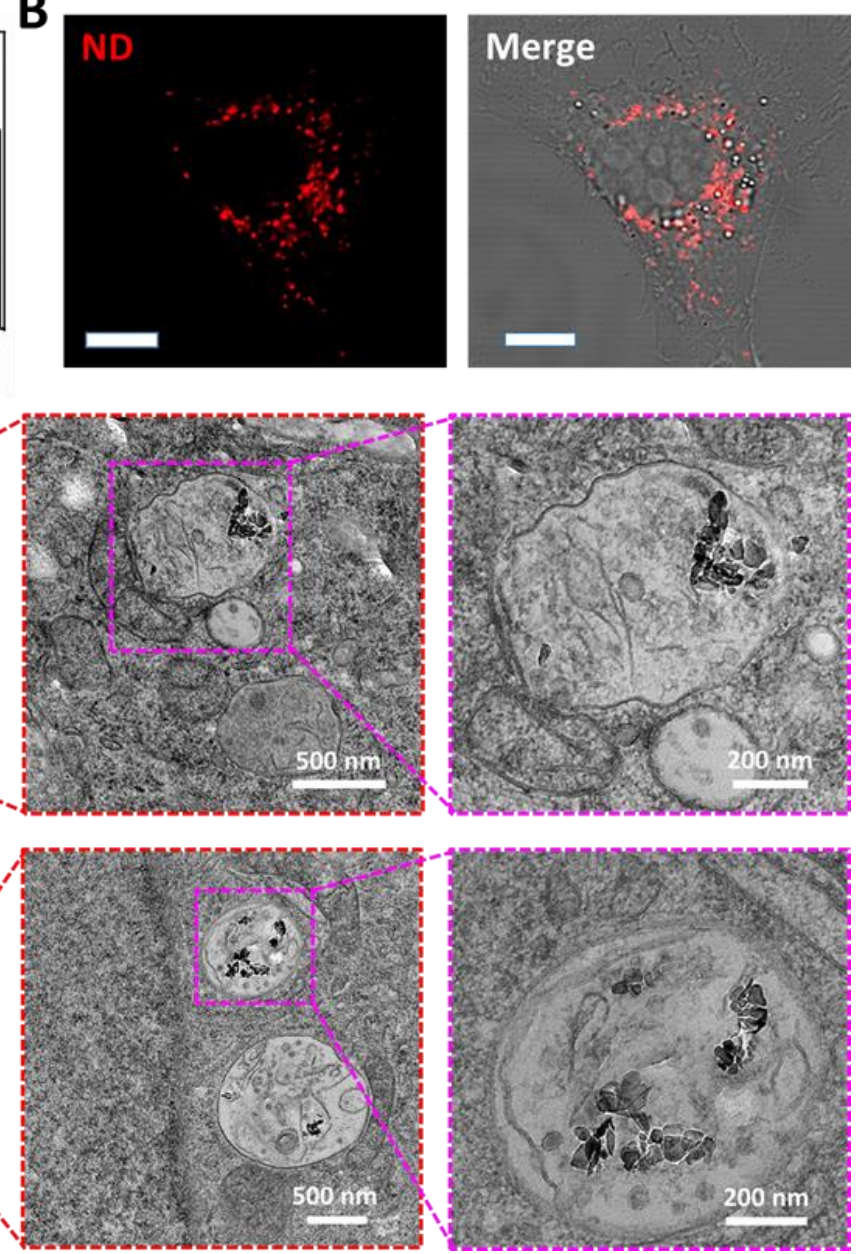

Figure 2. (A) Cell viability of HeLa cells after 4 hours incubation with ND-NG-ICG. (B) Confocal microscopy images of ND-NG-ICG which were taken up into HeLa cells at a concentration of $100 \mu \mathrm{g} / \mathrm{mL}$ after 4 hours (scale bar $=10 \mu \mathrm{m}$ ). (C) TEM images of ND-NGICG which were taken up into HeLa cells at a concentration of $10 \mu \mathrm{g} / \mathrm{mL}$ after 4 hours. (D) TEM images of ND-NG-ICG which were taken up into HeLa cells at a concentration of 100 $\mu \mathrm{g} / \mathrm{mL}$ after 4 hours.

\section{ND-NG-ICG-based temperature sensing at the nanoscale}

To measure the intracellular changes in the local temperature at the nanoscale under irradiation,

HeLa cells were cultured on a treated coverslip which was placed in a 12 well cell culture plate and incubated overnight. On the next day, fresh medium containing $10 \mu \mathrm{g} / \mathrm{mL}$ or $100 \mu \mathrm{g} / \mathrm{mL}$ 
of ND-NG or ND-NG-ICG was added. After 4 hours of incubation and 3x washing, cells were placed in a home-built confocal fluorescence microscope combined with optically detected magnetic resonance (ODMR) spectroscopy for measurement (Figure 3, Figure S5, S6 and S8). The zero field splitting $D_{0}$ of $\mathrm{NV}^{-}$center in ND is temperature dependent. Therefore, measurement of the zero field splitting via ODMR provides a robust readout of the local temperature at nanoscale. As shown in Figure 3B, the NDs are very bright compared to the autofluorescence of cell and the spots in cells were chosen for ODMR. The ODMR spectra of ND-NG-ICG under NIR irradiation were recorded and fitted with a double Lorentzian (Figure 3C). After 3 minutes of continuous irradiation, the ODMR spectrum shifted to the low frequency indicating a change of the temperature. However, ND-NG sample (Figure S7) revealed no significant shift in the ODMR spectra under NIR irradiation. To readout and monitor the intracellular changes in temperature, ODMR spectra were recorded up to 7 minutes continuously and the data was processed every 60 seconds. The ODMR spectra were fitted with a double Lorentzian function, and the changes in temperature were extracted according to the equation:

$$
\Delta T=\frac{\Delta D}{\alpha}
$$

where $\Delta D$ is the shift in the transition frequency and $\alpha=d D / d T=-74 \mathrm{kHz} / \mathrm{K}$ is the temperature susceptibility. ${ }^{27}$ In addition, the irradiation time over the change in temperature was fitted (Figure 3E). We found that the temperature increased sharply by more than $30{ }^{\circ} \mathrm{C}$ and saturated after approximately 250 seconds of irradiation for the ND-NG-ICG samples but there was no significant change in temperature for the ND-NG samples under irradiation. The result was independent of the concentration and similar local temperature increases were received for a ND-NG-ICG concentration of $10 \mu \mathrm{g} / \mathrm{mL}$ and $100 \mu \mathrm{g} / \mathrm{mL}$ (Figure 3E and Figure S8). 
The operating principle of thermometry using $\mathrm{NV}^{-}$centers relies on the accurate measurement of the transition frequency, which can be optically detected with high spatial resolution. For NV-based thermometry, the temperature sensitivity can be calculated the following equation:

$$
\eta_{E S R} \approx \frac{4}{3 \sqrt{3}} \frac{1}{d D / d T} \frac{\Delta f}{C \sqrt{R}}
$$

where $d D / d T$ is the NV temperature susceptibility, $\Delta f$ is the approximate ODMR linewidth, $C$ is ODMR contrast, and $R$ is the average photon counts. Applying $d D / d T=74 \mathrm{kHz} / \mathrm{K}, C \approx$ $0.05, \Delta f=5 \mathrm{MHz}$, and $R=3 \mathrm{M}$ cts/s resulted in a calculated sensitivity of $\eta=600 \mathrm{mK} / \sqrt{\mathrm{Hz}}$. Theoretically, a single $\mathrm{NV}^{-}$center can potentially exhibit a sensitivity higher than $1 \mathrm{mK} / \sqrt{\mathrm{Hz}}$.

\section{Temperature measurements at the macroscopic level}

To correlate the changes in temperature at the nanoscale to a macroscopic level, the photothermal effect of ND-NG-ICG was studied using a thermocouple in aqueous solution. The photothermal effect of ND-NG-ICG was measured under the same condition (810 nm lamp, $0.35 \mathrm{~W} / \mathrm{cm}^{2}$ ), which were applied during ODMR spectroscopy. Firstly, we evaluated the change in temperature of ND-NG in the absence of the ICG photothermal probe at different concentrations (Figure 3D) in aqueous solvent. A concentration-independent temperature increase of less than $2{ }^{\circ} \mathrm{C}$ was detected, which reached a saturation after 5 minutes of irradiation indicating that ND-NG did not possess photothermal activity. In contrast, free ICG and NDNG-ICG showed similar and pronounced concentration-dependent temperature increase. After 5 minutes of irradiation of free ICG, temperature changes of $11^{\circ} \mathrm{C}(10 \mu \mathrm{g} / \mathrm{mL}$ ICG $)$ up to $36{ }^{\circ} \mathrm{C}$ (200 $\mu \mathrm{g} / \mathrm{mL}$ ICG) were detected. Irradiation of ND-NG-ICG for 5 minutes induced temperature increase of $2{ }^{\circ} \mathrm{C}(10 \mu \mathrm{g} / \mathrm{mL}$ ND-NG-ICG with a loaded ICG content of $2.44 \mu \mathrm{g} / \mathrm{mL})$ up to $18{ }^{\circ} \mathrm{C}(200 \mu \mathrm{g} / \mathrm{mL}$ ND-NG-ICG with a loaded ICG content of $48.77 \mu \mathrm{g} / \mathrm{mL})$. These results indicate that temperature changes measured at the macroscopic level in bulk solvent are much 
lower than the temperature changes at the nanoscale probably due to diffusion paths of thermally activated molecules.

A

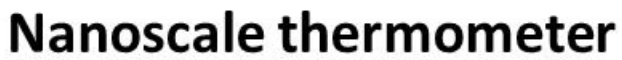

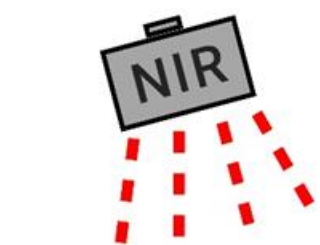

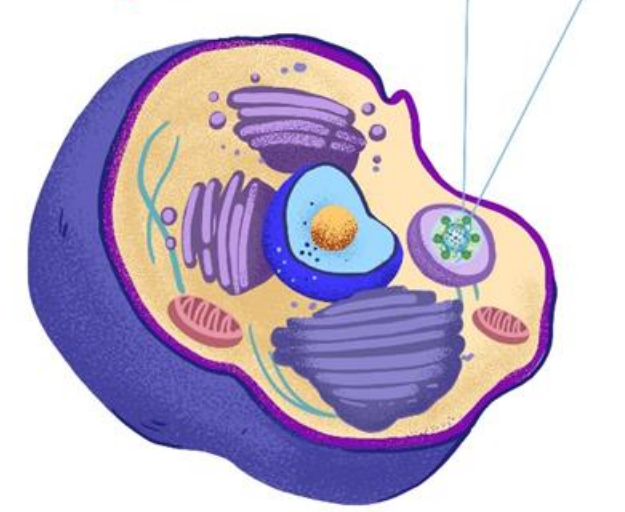

B

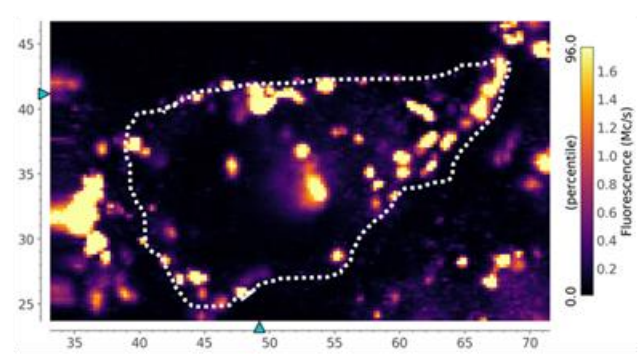

D

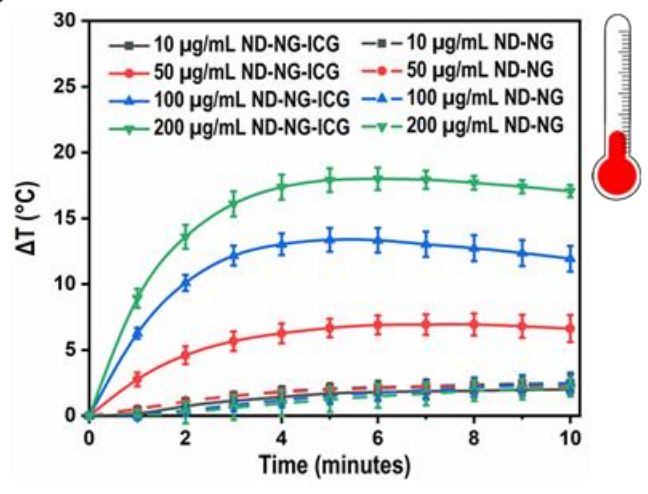

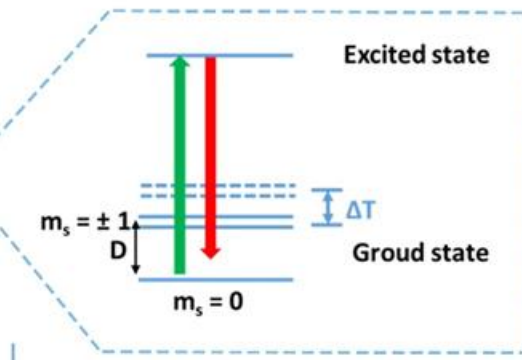

\section{Readout}

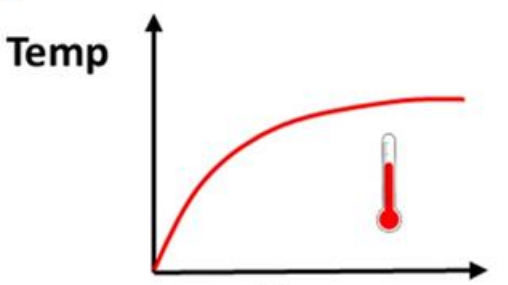

Time

C

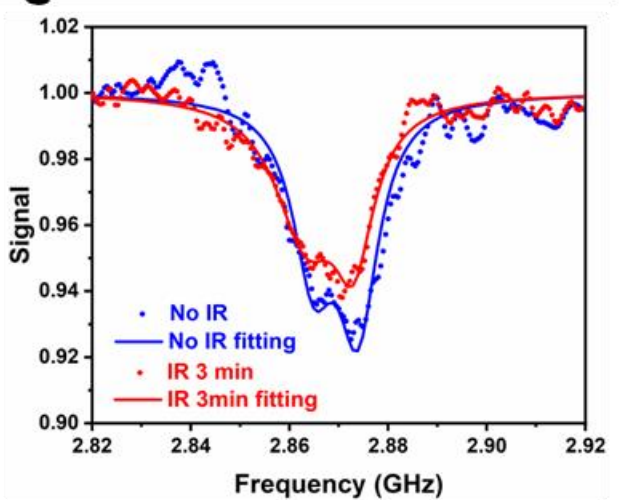

E

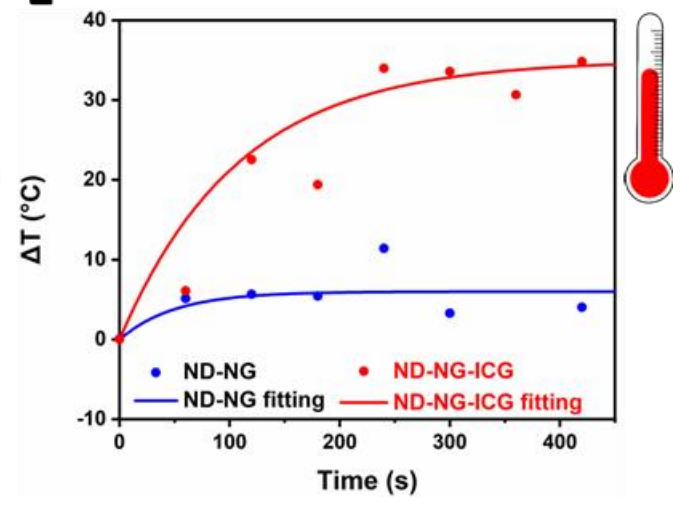


Figure 3. (A) Schematic presentation of the temperature measurements in living cells. Simplified energy levels diagram of the $\mathrm{NV}^{-}$center in ND displaying a ground state of spin triplet and an excited state. Under zero magnetic field, the $\mathrm{m}_{\mathrm{s}}= \pm 1$ sub-levels are split from the $\mathrm{m}_{\mathrm{s}}=0$ state by a temperature-dependent zero field splitting $D_{0}$. The intracellular change in temperature can be monitored by the shift of the zero-field splitting parameter $D_{0}$ using ODMR spectroscopy. At room temperature, $D_{0} \approx 2.87 \mathrm{GHz}$ and $D_{0}$ varies depending on temperature $\mathrm{T}$ as $\Delta T=\Delta D / \alpha$, where $\alpha=-74 \mathrm{kHz} / \mathrm{K}$. (B) Fluorescence image of ND-NG-ICG in a living cell, after 4 hours of incubation using a ND-NG-ICG concentration of $10 \mu \mathrm{g} / \mathrm{mL}$. (dash line is the cell boarder). (C) Representative ODMR spectra of ND-NG-ICG which was fitted with a double Lorentzian under near-infrared (NIR) irradiation (810 nm lamp; $\left.0.35 \mathrm{~W} / \mathrm{cm}^{2}\right)$ at 0 minute and 3 minutes, after 4 hours of incubation using a ND-NG-ICG concentration of 10 $\mu \mathrm{g} / \mathrm{mL}$. (D) The thermal profile of ND-NG and ND-NG-ICG at a concentration of $10 \mu \mathrm{g} / \mathrm{mL}$, $50 \mu \mathrm{g} / \mathrm{mL}, 100 \mu \mathrm{g} / \mathrm{mL}$, and $200 \mu \mathrm{g} / \mathrm{mL}$ under near-infrared (NIR) irradiation (810 $\mathrm{nm}$ lamp;

$0.35 \mathrm{~W} / \mathrm{cm}^{2}$ ). (E) The change of intracellular temperature measured by ODMR for NG-NGICG and ND-NG over 420 seconds under near-infrared (NIR) irradiation (810 nm lamp; 0.35 $\mathrm{W} / \mathrm{cm}^{2}$ ), after 4 hours of incubation using a ND-NG-ICG concentration of $10 \mu \mathrm{g} / \mathrm{mL}$.

\section{ND-NG-ICG serves as temperature reporting heating source displaying a pronounced photothermal effect in live cells}

To understand the nanoscale photothermal effects on cells, LED light with a wavelength of 810 $\mathrm{nm}$ was focused onto the sample area through the objective of a microscope for 20 minutes. The light power density was set to $0.35 \mathrm{~W} / \mathrm{cm}^{2}$, which was the same value we used to measure the local temperature by ODMR spectroscopy. After 20 minutes of irradiation, cells were further incubated for 4 hours and analyzed by live/dead staining. As shown in Figure 4B, without irradiation at $810 \mathrm{~nm}$, cells could proliferate well, showed a normal cell morphology, and almost all cells remained viable after incubation with $10 \mu \mathrm{g} / \mathrm{mL}$ or $100 \mu \mathrm{g} / \mathrm{mL}$ of ND-NG- 
ICG. After irradiation at $810 \mathrm{~nm}$, the cell viability was not impaired for low concentrations of ND-NG-ICG (10 $\mu \mathrm{g} / \mathrm{mL})$ and ND-NG (Figure S9). However, most of the cells treated with higher concentration of ND-NG-ICG $(100 \mu \mathrm{g} / \mathrm{mL})$ were found dead in the live/dead imaging strictly within the irradiated area. The similar influence was convince by cell early apoptosis experiments as well (Figure S10).

Based on the ODMR measurements, we recorded the local temperature change $(\Delta T)$ inside cells. Remarkably, temperature changes of not less than $30{ }^{\circ} \mathrm{C}$ after only 250 seconds of irradiation at $810 \mathrm{~nm}$ for low concentrated ND-NG-ICG $(10 \mu \mathrm{g} / \mathrm{mL})$ and high concentrated ND-NG-ICG $(100 \mu \mathrm{g} / \mathrm{mL})$ were detected. Surprisingly, the local temperature increase is independent of the nanoparticle concentrations, because both $10 \mu \mathrm{g} / \mathrm{mL}$ and $100 \mu \mathrm{g} / \mathrm{mL}$ revealed a similar increase in local temperature after heating (Figure 3E and Figure S8). However, no cell death was observed for low concentrated ND-NG-ICG (10 $\mu \mathrm{g} / \mathrm{mL})$ under irradiation at $810 \mathrm{~nm}$ for 20 minutes. We propose that cells could compensate a pronounced local temperature increase if only few heating sources of low concentrated ND-NG-ICG are present. In this way, our results are in line with other reports i.e. on mitochondria, which can tolerate comparatively high temperatures without effecting cell viability. ${ }^{10}$ In contrast, the presence of many local ND-NG-ICG heating sources $(100 \mu \mathrm{g} / \mathrm{mL})$ produced heat over a larger volume within the cellular vesicles, which induced the observed cell death. 


\section{A Nanoscale heater for cell death manipulation}
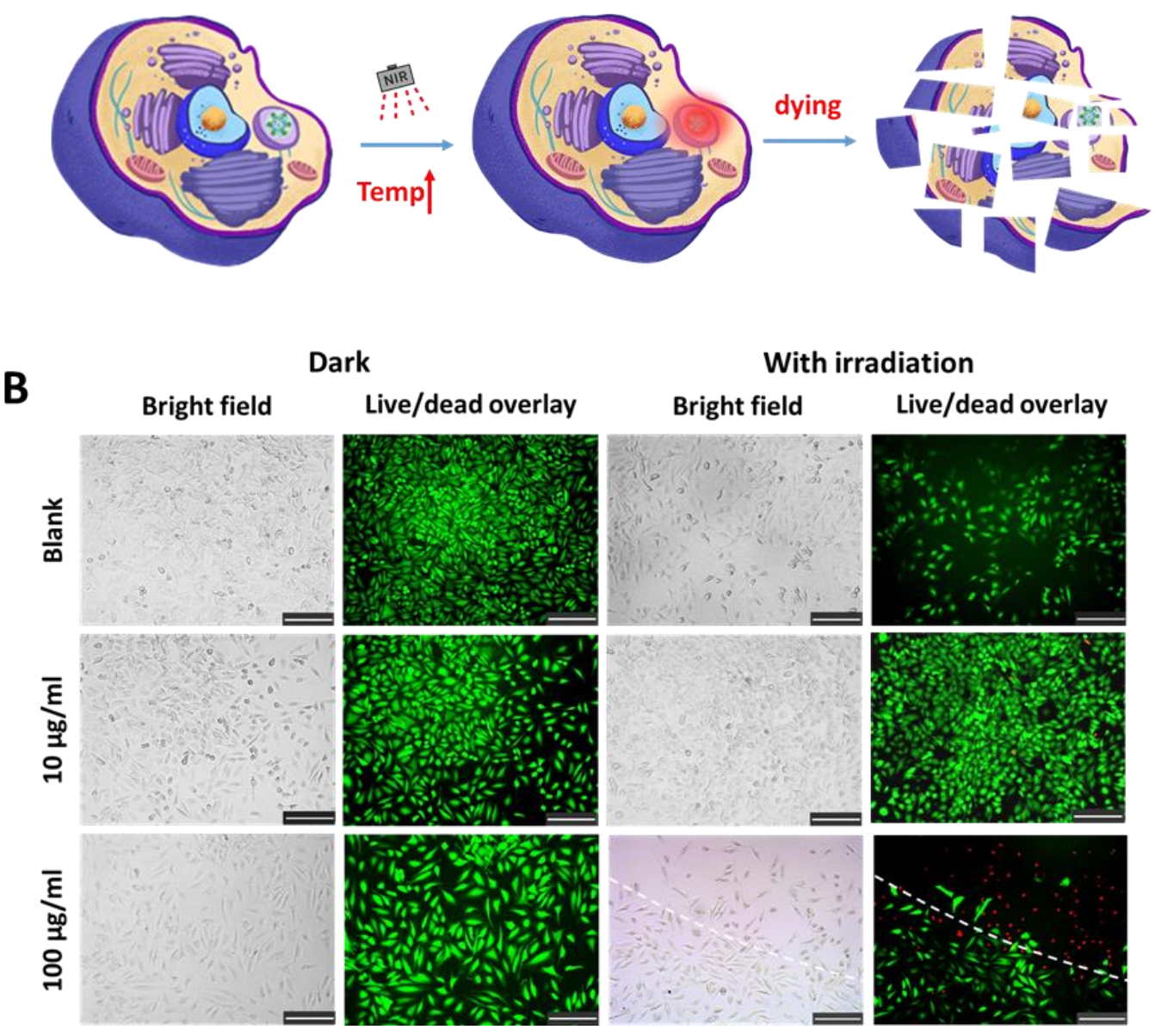

Dark

With irradiation

Figure 4. (A) Sketch of nanoscale heater for cell death manipulation. (B) Live/dead staining of HeLa cells incubated at different concentration of ND-NG-ICG after 20 minutes irradiation using a near-infrared (NIR) LED lamp (810 nm lamp; $0.35 \mathrm{~W} / \mathrm{cm}^{2}$; scale bar $\left.=200 \mu \mathrm{m}\right)$. Green and red color represent live and dead cells, respectively.

\section{Conclusion}

In summary, we have prepared the first local heating and temperature self-reporting photothermal agent with pronounced bioactivity. This nanoscale sensor was able to monitor the temperature increase after heating due to the photothermal effects in situ and in living cells. The ODMR results showed an increase of the local temperature inside endosomes of not less than $30^{\circ} \mathrm{C}$ within 250 seconds of irradiation using a concentration of ND-NG-ICG of $10 \mu \mathrm{g} / \mathrm{mL}$ 
and $100 \mu \mathrm{g} / \mathrm{mL}$. Obviously, cells could tolerate a high local temperature increase if the concentration of the photothermal agent is low. This data supports that the intracellular temperature can be inhomogeneous and can even differ by $30^{\circ} \mathrm{C}$ without affecting cell viability. Our study investigated the local temperature change induced by a photothermal agent, which has not been revealed by former studies on PTT. This work helps to gain a deeper understanding of the photothermal effect at the nanoscale and opens the way for further studies on photothermal effect in different intracellular environments and its effect on cell viability. Furthermore, it has been reported that a local temperature change can influence the cell division time recently ${ }^{28}$. An ND-based nanothermometer could represent a valuable tool to investigate temperature-driven biological processes in more detail intracellularly or even at the subcellular level.

\section{Methods}

\section{Preparation of ND-NG}

Firstly, $100 \mu \mathrm{L} \mathrm{ND}(2 \mathrm{mg} / \mathrm{mL})$ stock solution was dispersed in $300 \mu \mathrm{L}$ water. Afterwards, 100 $\mu \mathrm{L}$ PVP (20 mg/mL), $200 \mu \mathrm{L}$ PEI (5 mg/mL), and $300 \mu \mathrm{L}$ PBS buffer (10 mM, pH 7.4) were added. After 5 minutes sonication, 2 mg of 4-arm PEG-SCM was added to cross-link the PEI on the surface of ND. The final volume was adjusted to $1 \mathrm{~mL}$ with MilliQ water. After sonication for 30 minutes and reaction for 90 minutes on a shaker at $800 \mathrm{rpm}$, the obtained NDNGs were washed 3 times by centrifugation at $12,000 \mathrm{~g}$ for 20 minutes.

\section{Preparation of ND-NG-ICG}

$100 \mu \mathrm{L}$ of a $2 \mathrm{mg} / \mathrm{mL}$ ND-NG solution was mixed with $400 \mu \mathrm{L}$ of $0.1 \mathrm{mg} / \mathrm{mL}$ ICG and was sonicated 30 seconds, then reacted overnight on a shaker at $800 \mathrm{rpm}$, the ND-NG-ICG were obtained by centrifugation at $12,000 \mathrm{~g}$ for 20 minutes to remove the supernatant. 


\section{Loading efficiency}

A standard curve of ICG absorbance was prepared to calculate the ICG content in ND-NGICG as followed: a $20 \mu \mathrm{L}$ ICG solution with concentration of $62.5 \mu \mathrm{g} / \mathrm{mL}, 31.25 \mu \mathrm{g} / \mathrm{mL}, 15.63$ $\mu \mathrm{g} / \mathrm{mL}, 7.81 \mu \mathrm{g} / \mathrm{mL}, 3.91 \mu \mathrm{g} / \mathrm{mL}, 1.95 \mu \mathrm{g} / \mathrm{mL}$ water, and supernatant of ND-NG-ICG were added in separate wells of 384-well low volume well plate. And then absorbance intensity at $789 \mathrm{~nm}$ were obtained by a Tecan Spark 20M. The absorbance intensity-concentration curve was drawn and the ICG content in ND-NG-ICG was calculated.

\section{ICG releasing curve}

$200 \mu \mathrm{g}$ ND-NG-ICG was added to $500 \mu \mathrm{L}$ MilliQ water and put on a shaker at $800 \mathrm{rpm}$, at the setting time points, one of the ND-NG-ICG aliquots was centrifuged at 12,000 $\mathrm{g}$ for 20 minutes, the absorbance of supernatant at $789 \mathrm{~nm}$ was measured by a Tecan Spark 20M, and the released ICG content was calculated.

\section{UV-Vis Absorbance and Fluorescence}

$20 \mu \mathrm{L}$ of ICG solution $(0.1 \mathrm{mg} / \mathrm{mL})$ were prepared in separate wells of a 384 -well low volume microplate. Absorbance scans from 200 to $1000 \mathrm{~nm}$ were obtained using a Tecan Spark 20M microplate reader. And the emission spectra of ICG was measured using a $40 \mu \mathrm{L}$ micro cuvette at Perkin Elmer Lambda 900 device. It was excited at $600 \mathrm{~nm}$ and the emission spectra was collected from $750 \mathrm{~nm}$ to $1000 \mathrm{~nm}$. And the spectra were normalised.

\section{Transmission Electron Microscopy (TEM)}

One drop of a $0.1 \mathrm{mg} / \mathrm{mL}$ solution of ND-NGs in MilliQ was placed onto an oxygen treated copper grid. A Jeol 1400 transmissions electron microscope was used to obtain bright field images. 


\section{Dynamic Light Scattering (DLS)}

$500 \mu \mathrm{L}$ of $0.1 \mathrm{mg} / \mathrm{mL}$ solutions of ND, ND-NG, ND-NG-ICG in MilliQ were transferred into a borosilicate glass cuvette. They was measured at $25^{\circ} \mathrm{C}$ with a $90^{\circ}$ angle using a particle sizer (Malvern Zetasizer Nano-S90 (Nano series)). The hydrodynamic diameter distribution was presented as intensity. Zeta potential was measured at $25{ }^{\circ} \mathrm{C}$ with a Malvern Zetasizer NanoS90 (Nano series).

Photothermal profile study for bulk ND-NG, ND-NG-ICG and pure ICG solutions in water

Various concentration of ND-NG, ND-NG-ICG and pure ICG were prepared to measure the photothermal temperature changes. Subsequently, a K-type thermocouple wire and an automated temperature input device (USB-TC01, National Instruments) were employed to record the real-time temperature change of $100 \mu \mathrm{L}$ of each samples. The process were repeated three times for each concentration. An $810 \mathrm{~nm}$ LED lamp (Thor Labs, M810L3) was used to irradiate the samples, light irradiance was $0.35 \mathrm{~W} / \mathrm{cm}^{2}$.

\section{Samples preparation of cell with ND-NG-ICG for Transmission Electron Microscopy} (TEM)

Cells were cultured in a $24-w e l l$ plate pre-placed with carbon coated sapphire discs $(\mathrm{d}: 3 \mathrm{~mm})$ with a density of 50,000 cells/mL. After co-incubation with nanoparticles (NPs), sapphire discs were placed between two aluminum plates to create a 'sandwich' and afterwards were mounted into a holder (Engineering Office, M. Wohlwend) and immediately fixated in a Wohlwend 
HPF Compact 01 high-pressure freezer (Engineering Office, M. Wohlwend) with a pressure of 2100 bar. The frozen samples were then stored in liquid nitrogen.

Frozen sapphire discs were carefully removed from the aluminum 'sandwich' and transferred into $1 \mathrm{~mL}$ pre-cooled freeze substitution medium $(0.2 \%(\mathrm{w} / \mathrm{v})$ osmium tetroxide, $0.1 \%(\mathrm{w} / \mathrm{v})$ uranyl acetate, $5 \%(\mathrm{v} / \mathrm{v})$ distilled water in acetone) and kept in a freeze substitution unit (AFS2,Leica). Samples were then slowly warmed up to $0{ }^{\circ} \mathrm{C}$ over a period of 20 hours in the unit. After being warmed up, the freeze-substituted samples were brought to room temperature, then the substitution medium was removed and the discs were washed three times with acetone at half an hour intervals. Then the discs were infiltrated sequentially in gradient epoxy resinacetone mixture $(1 ; 1,1: 2$, and $2: 1)$ for $1 \mathrm{~h}$. Samples were then infiltrated in $100 \%$ epoxy resin overnight. Finally, each sample was transferred into a new Eppendorf tube containing fresh epoxy resin for polymerization at $60{ }^{\circ} \mathrm{C}$ for $24 \mathrm{~h}$.

Following polymerisation, sapphire discs were detached using liquid nitrogen. Resin blocks with the cells imprinted on were trimmed and sectioned into $100 \mathrm{~nm} / 80 \mathrm{~nm}$ sections by a $45^{\circ}$ diamond knife (Diatome) in EM UC6 ultramicrotome (Leica).

\section{Confocal Microscopy}

Confocal laser scanning microscopy (CLSM) was utilized to observe the cellular uptake of ND-NG-ICG. HeLa cells were seeded in an ibidi 8 well $\mu$-slide $\left(10^{5}\right.$ cells $/ \mathrm{mL}, 200 \mu \mathrm{L}$ each well) and incubated overnight. The cells were washed once with Dulbecco's phosphatebuffered saline (DPBS) and $100 \mu \mathrm{g} / \mathrm{mL}$ ND-NG-ICG (mixed with cell culture medium) was added to the cells. After four hour incubation at $37{ }^{\circ} \mathrm{C}$, the cells were washed three times with DPBS to remove the particles which were not taken up, then fresh medium was added. A Leica TCS SP5 confocal microscope system was used to obtain the microscopy images and later they were analyzed with ImageJ software. 


\section{Cytotoxicity study}

HeLa cells were seeded on a white 96 well half-area flat bottom cell culture microplate (Greiner). Seeded cell concentration was 110,000 cell $/ \mathrm{mL}, 50 \mu \mathrm{L}$ in each well. After incubation at $37{ }^{\circ} \mathrm{C}$ overnight and $5 \% \mathrm{CO}_{2}$, various concentrations of ND-NG-ICG were added to the cells and incubated again for 24 hours. The next day, the cells were washed three times with DPBS and fresh cell culture medium (containing DMEM with 10\% FBS, 1\% MEM NEAA, and 1\% PenStrep) was added. Afterward, freshly prepared CellTiter-Glo luminescent cell viability assay (Promega) was applied as instructed by the manufacturer's protocol. The luminescence signals were recorded with a Promega GloMax multi detection plate reader.

\section{Sample preparation for intracellular temperature measurements}

Coverslips of $15 \mathrm{~mm}$ diameter were placed in a 12 well cell culture plate to seed HeLa cells. The cell concentration was $10^{6}$ cells $/ \mathrm{mL}, 500 \mu \mathrm{L}$ in each well. After overnight incubation, 10 $\mu \mathrm{g} / \mathrm{mL}$ ND-NG and ND-NG-ICG samples in cell culture medium were added to the cells. Subsequently after four hour incubation at $37{ }^{\circ} \mathrm{C}$, the cells were washed 3 times with DPBS to remove the excess nanoparticles which were not uptaken, and fresh clear DMEM (without phenol red) was added to the cells. The ODMR measurements were performed immediately. As depicted in Figure S5, the cell attached coverslip was put on ODMR sample holder and a sandwich-like formation was secured, where the microwave antenna and the cells were in the middle of two coverslips which was filled with cell culture medium (Figure S6).

\section{Live/dead staining of HeLa cell}


HeLa cells were seeded on two ibidi 8 well $\mu$-slide with a cell concentration of 160,000 cells $/ \mathrm{mL}(300 \mu \mathrm{L}$ in each well) and incubated overnight. Subsequently $10 \mu \mathrm{g} / \mathrm{mL}$ and 100 $\mu \mathrm{g} / \mathrm{mL}$ ND-NG and ND-NG-ICG samples were prepared by diluting the stock solution with cell culture medium. $200 \mu \mathrm{L}$ of each concentration of samples were added to the cells and kept in the incubator for four hours. Then both plates were washed three times with DPBS, and added fresh culture medium. Meanwhile, the $810 \mathrm{~nm}$ LED was mounted to a Leica DMi8 microscope using a microscope collimation adapter (Thor Labs) to irradiate one of the treated $\mu$-slides for 20 minutes $\left(0.35 \mathrm{~W} / \mathrm{cm}^{2}\right)$. An incubator was used during the irradiation to keep the cells in $37{ }^{\circ} \mathrm{C}$ to simulate human body temperature. After photo-treating the cells, both $\mu$ slides were kept in an incubator for additional four hours. Afterward, cells were treated with freshly prepared live/dead staining solution. The solution was prepared as stated in the ibidi protocol. Briefly $8 \mu \mathrm{L}$ fluorescein diacetate $(5 \mathrm{mg} / \mathrm{mL})$ and $100 \mu \mathrm{L}$ of propidium iodide (1 $\mathrm{mg} / \mathrm{mL}$ ) were mixed with DPBS (5 mL). $300 \mu \mathrm{L}$ of live/dead staining solution was added to each well of the $\mu$-slide and kept in dark for five minutes. Then, the cells were washed with DPBS. Imaging was performed immediately using a Leica DMi8 microscope with a Leica MC170 HD camera system.

\section{Early apoptosis detection with Annexin V staining}

HeLa cells were grown in a 10 well CELLview Greiner Bio-One cell culture slide $\left(10^{6}\right.$ cells $/ \mathrm{mL}$, $100 \mu \mathrm{L}$ in each well). Next day, $100 \mu \mathrm{g} / \mathrm{mL}$ ND-NG-ICG in cell culture medium was added to some of the wells (100 $\mu \mathrm{L}$ each). After 4 hours of incubation, cells were washed with DPBS and then treated with $810 \mathrm{~nm}$ light same way as earlier mentioned in live/dead staining of the HeLa cells. Then again after 4 hours incubation, eBioscience ${ }^{\mathrm{TM}}$ Annexin V-FITC apoptosis detection kit was used to stain the cells simultaneously with propidium iodide (PI). Stock 10x 
binding buffer from the assay kit was diluted to $1 \mathrm{x}$. For each $200 \mu \mathrm{L}$ diluted binding buffer, 5 $\mu \mathrm{L}$ of Annexin V-FITC and $5 \mu \mathrm{L}$ PI were mixed, then $100 \mu \mathrm{L}$ of staining solution was added to each well and left in dark for 15 minutes at room temperature. After 15 minutes, the staining solution was replaced with $1 \mathrm{x}$ binding buffer and immediately the microscopy was performed.

\section{Conflicts of interest}

The authors declare no competing financial interest.

\section{Supporting Information}

Supporting Information is available from the $* * * * *$ or from the author.

\section{Acknowledgements}

The authors thank Mr. Kai Philipps for the ICG emission spectrum measurement, Christoph Sieber for the preparation TEM samples of cells, and Dr. David Yuen Wah Ng for the fruitful discussion and suggestions. The authors are grateful for the financial support from the European Union's Horizon 2020 Research and Innovation Program under FETOPEN grant agreement no. 858149 (AlternativeToGd) and from the Deutsche Forschungsgemeinschaft (DFG, German Research Foundation) - Project number 316249678 - SFB 1279 (C04) and SFB 1279 (C02) as well as VW Stiftung. Y. Wu thanks the China Scholarship Council for a fellowship.

\section{Author Contributions}

† Yingke Wu and Md Noor A Alam contributed equally. Y.W., M.N.A.A., M.R., and T.W. initiated this project. Y.W. prepared the materials, performed material characterizations, 
performed the TEM measurement of nanodiamond in cells, and partially performed ODMR measurements. M.N.A.A. performed photothermal profile study, prepared cells for confocal microscopy and intracellular temperature measurement, cytotoxicity study, live/dead and Annexin-V staining of the cells. M.R. performed confocal microscopy and prepared cells for TEM. P.B. performed ODMR measurements and analyzed the data. A.E. performed some of the initial ODMR measurements. S.F. and H.B. designed, supplied, and cultured the cells for ODMR measurements. M.W. discussed the concept and results in the projects. Y.W. prepared the draft of the manuscript. Y.W, M.N.A.A., M.R., and P.B. prepared the final manuscript based on discussions with all authors. M.R., T.W., and F.J. supervised the project.

\section{References}

1 Franceschi, C. Cell proliferation, cell death and aging. Aging Clin. Exp. Res. 1, 3-15 (1989).

2 Marsden, H., Crombie, I. K. \& Subak-Sharpe, J. Control of protein synthesis in herpesvirusinfected cells: analysis of the polypeptides induced by wild type and sixteen temperaturesensitive mutants of HSV strain 17. J. Gen. Virol. 31, 347-372 (1976).

3 Martinez, J., Georgoff, I. \& Levine, A. Cellular localization and cell cycle regulation by a temperature-sensitive p53 protein. Genes Dev. 5, 151-159 (1991).

4 Seymour, R. S. Biophysics and physiology of temperature regulation in thermogenic flowers. Biosci. Rep. 21, 223-236 (2001).

5 Wei, W., Zhang, X., Zhang, S., Wei, G. \& Su, Z. Biomedical and bioactive engineered nanomaterials for targeted tumor photothermal therapy: a review. Mater. Sci. Eng.C 104, 109891 (2019).

6 Doughty, A. C. et al. Nanomaterial applications in photothermal therapy for cancer. Materials 12, 779 (2019).

$7 \quad$ Hsiao, C.-W. et al. Photothermal tumor ablation in mice with repeated therapy sessions using NIR-absorbing micellar hydrogels formed in situ. Biomaterials 56, 26-35 (2015).

8 Chen, Q. et al. Photothermal therapy with immune-adjuvant nanoparticles together with checkpoint blockade for effective cancer immunotherapy. Nat. Commun. 7, 1-13 (2016).

9 Zhang, C. et al. A polyoxometalate cluster paradigm with self-adaptive electronic structure for acidity/reducibility-specific photothermal conversion. J. Am. Chem. Soc. 138, 8156-8164 (2016).

10 Chrétien, D. et al. Mitochondria are physiologically maintained at close to 50 C. PLoS Biol. 16, e2003992 (2018).

11 Chen, F. \& Cai, W. Nanomedicine for targeted photothermal cancer therapy: where are we now? Nanomed. 10, 1-3 (2015).

12 Norouzi, H., Khoshgard, K. \& Akbarzadeh, F. In vitro outlook of gold nanoparticles in photothermal therapy: a literature review. Lasers Med. Sci. 33, 917-926 (2018).

13 Matsumura, Y. \& Maeda, H. A new concept for macromolecular therapeutics in cancer chemotherapy: mechanism of tumoritropic accumulation of proteins and the antitumor agent smancs. Cancer Res. 46, 6387-6392 (1986).

14 Jaque, D. et al. Nanoparticles for photothermal therapies. Nanoscale 6, 9494-9530 (2014). 
15 Yang, J.-M., Yang, H. \& Lin, L. Quantum dot nano thermometers reveal heterogeneous local thermogenesis in living cells. ACS Nano 5, 5067-5071 (2011).

16 Okabe, K. et al. Intracellular temperature mapping with a fluorescent polymeric thermometer and fluorescence lifetime imaging microscopy. Nat. Commun. 3, 1-9 (2012).

17 Kiyonaka, S. et al. Genetically encoded fluorescent thermosensors visualize subcellular thermoregulation in living cells. Nat. Methods 10, 1232-1238 (2013).

18 Yang, F. et al. Measurement of local temperature increments induced by cultured HepG2 cells with micro-thermocouples in a thermally stabilized system. Sci. Rep. 7, 1-11 (2017).

19 Maze, J. R. et al. Nanoscale magnetic sensing with an individual electronic spin in diamond. Nature 455, 644-647 (2008).

20 Sekiguchi, T., Sotoma, S. \& Harada, Y. Fluorescent nanodiamonds as a robust temperature sensor inside a single cell. Biophys. Physicobiol. 15, 229-234 (2018).

21 Neumann, P. et al. High-precision nanoscale temperature sensing using single defects in diamond. Nano Lett. 13, 2738-2742 (2013).

22 Kucsko, G. et al. Nanometre-scale thermometry in a living cell. Nature 500, 54-58 (2013).

23 Simpson, D. A. et al. Non-neurotoxic nanodiamond probes for intraneuronal temperature mapping. ACS Nano 11, 12077-12086 (2017).

24 Plakhotnik, T., Doherty, M. W., Cole, J. H., Chapman, R. \& Manson, N. B. All-optical thermometry and thermal properties of the optically detected spin resonances of the NVcenter in nanodiamond. Nano Lett. 14, 4989-4996 (2014).

25 Tsai, P. C. et al. Measuring nanoscale thermostability of cell membranes with single golddiamond nanohybrids. Angew. Chem. Int. Ed. 56, 3025-3030 (2017).

$26 \mathrm{Wu}, \mathrm{Y}$. et al. Fluorescent Nanodiamond-Nanogels for Nanoscale Sensing and Photodynamic Applications. https://doi.org/10.26434/chemrxiv.13317494.v2.

27 Acosta, V. M. et al. Temperature dependence of the nitrogen-vacancy magnetic resonance in diamond. Phys. Rev. Lett. 104, 070801 (2010).

28 Choi, J. et al. Probing and manipulating embryogenesis via nanoscale thermometry and temperature control. Proc. Natl. Acad. Sci. U. S. A.117 14636-14641 (2020). 